\title{
Continuous Stirred Tank Reactor Optimisation via Simulated Annealing, Firefly and Ant Colony Optimisation Elements on the Steepest Ascent
}

\author{
Pongchanun Luangpaiboon
}

\begin{abstract}
The objective of this work is to make use of conventional response surface methodologies and basic elements from metaheuristic algorithms in the design of influential variables for engineering systems. A method of steepest ascent and its integrated approaches with simulated annealing, firefly and ant colony optimisation algorithms, are compared on a simulated continuous stirred tank reactor or CSTR with various levels of signal noise. These metaheuristics contain the complicatedness in terms of their parameters. An additional series of computational experiments were conducted and analysed in terms of the minimax and mean squared error performance measures including Taguchi's signal to noise ratio. Proper levels of these parameters are analysed to recommend the best parameter choices. On the experimental results of all the algorithms with the preferable levels of parameters, the method of steepest ascent seems to be the most efficient on the CSTR surface at the lower levels of noise. However, the integrated approaches with all simulated annealing, firefly and ant colony optimisation elements work well when the standard deviation of the noise is at higher levels. Although the average, the standard deviation of the greatest actual concentration of the product and percentage of sequences ended at the optimum from the integrated algorithms with simulated annealing and ant colony optimisation seem to be better, they need more average design points, especially with ant colony optimisation element, to converge to the optimum when compared.
\end{abstract}

Index Terms-Simulated annealing, firefly, ant colony optimisation; steepest ascent; continuous stirred tank reactor.

\section{INTRODUCTION}

The objective of response surface methodology is to describe how the response of a process varies with changes in $k$ predictor variables. Estimation of such surfaces, and hence identification of near optimal settings for process variables is an important practical issue with interesting theoretical aspects. The predictor variables determined will depend on the specific field of the application. Most industrial processes have some predictor variables. These predictor variables can be adjusted by plant operators or by automatic control mechanisms to enhance the efficiency of the machine. One among those processes of chemical reactor is the most

Manuscript received March 21, 2011; This work was supported by the National Research University Project of Thailand, Office of Higher Education Commission. The author wishes to thank the Faculty of Engineering, Thammasat University, THAILAND for the financial support.

P. Luangpaiboon is an Associate Professor, the Industrial Statistics and Operational Research Unit (ISO-RU), Department of Industrial Engineering, Faculty of Engineering, Thammasat University, 12120, THAILAND. [Phone: (662)564-3002-9; Fax: (662)564-3017; e-mail lpongch@engr.tu.ac.th]. influential and therefore important unit to be encountered by a chemical engineer.

Non-linear and linear system descriptions are derived. A type of reactor widely used in various industries is a well-stirred tank into which there is a continuous flow of reacting material and from which the reacted or partially reacted material passes continuously. To ensure the successful operation of a continuous stirred tank reactor or CSTR it is necessary to understand its dynamic characteristics. The aim of this paper is to introduce some basic concepts of chemical reaction system modelling and develop computer simulation models for the CSTR. A good understanding will ultimately enable effective control systems design via its predictor variables.

There is much current interest in optimisation methods with the stochastic element. Natural intelligence-inspired approximation optimisation techniques called metaheuristics are then introduced. Moreover, metaheuristics have been used to avoid being trapped in local optima with a poor value [1]. The common factor in metaheuristics is that they combine rules and randomness to imitate natural phenomena. They widely grow and apply to solve many types of problems. The major reason is that metaheuristic approaches can guide the stochastic search process to iteratively seek near optimal solutions in practical and desirable computational time. Their properties expose useful information and overcome the large and noisy systems.

These algorithms are then received more attention in the last few decades. They can be categorised into three groups: biologically-based inspiration, e.g. genetic algorithm or GA [2], memetics algorithm or MAs [2], shuffled frog leaping algorithm or SFLA [2], firefly algorithm or FFA [3], bees algorithm or BEES [4], harmony search algorithm or HSA [5], neural network or NN [6], ant colony optimisation or ACO [7], evolutionary programming or EP [8], differential evolution or DE [9] and particle swarm optimisation or PSO [10]. Moreover, there are some with the socially-based inspiration, e.g. tabu search or TS [11] and the physically-based inspiration such as simulated annealing or SA [12].

The objective of this study is to compare the efficiency of sequential algorithms for on-line optimisation of a chemical process in the presence of noises. The method of steepest ascent and the integrated approach between the method of steepest ascent and three classes of metaheuristics, simulated annealing, firefly and ant colony optimisation algorithms, are selected and implemented on the CSTR. The context is maximising the concentration of a desired product of a 
chemical reactor with respect to feed rate, concentration and temperature. The paper is organised as follows. Section II is concerned with the related methods. Section III briefly discusses the main features of chemical reactors and, in particular, focuses on the dynamic model of CSTR. Sections $\mathrm{IV}$ and $\mathrm{V}$ are concerned with parameter settings for all three metaheuristics and the details of proposed methods, respectively. Section VI shows experimental results and analyses for comparing the performance of the proposed methods. Discussions and recommendations are also included and it is followed by acknowledgment and references.

\section{RELATED METHODS}

\section{A. Steepest Ascent Method (SAM)}

The procedure of SAM is that a hyperplane is fitted to the results from the initial $2^{k}$ factorial designs. The data from these design points are analysed. If there is an evidence of main effect(s), at some chosen level of statistical significance and no evidence of curvature, at the same level of significance, the direction of steepest ascent on the hyperplane is then determined by using principles of least squares and experimental designs. The next run is carried out at a point, which has some fixed distance in this direction, and further runs are carried out by continuing in this direction until no further increase in yield is noted [1].

When the response first decreases and no improvement of two more verified yields, another $2^{k}$ factorial design will be carried out, centered on the preceding design point. A new direction of steepest ascent is estimated from this latest experiment. Provided at least one of the coefficients of the hyperplane is statistically significantly different from zero, the search continues in this new direction (Figure 1). Once the first order model is determined to be inadequate, the area of optimum is identified via a second order model or a finishing strategy.

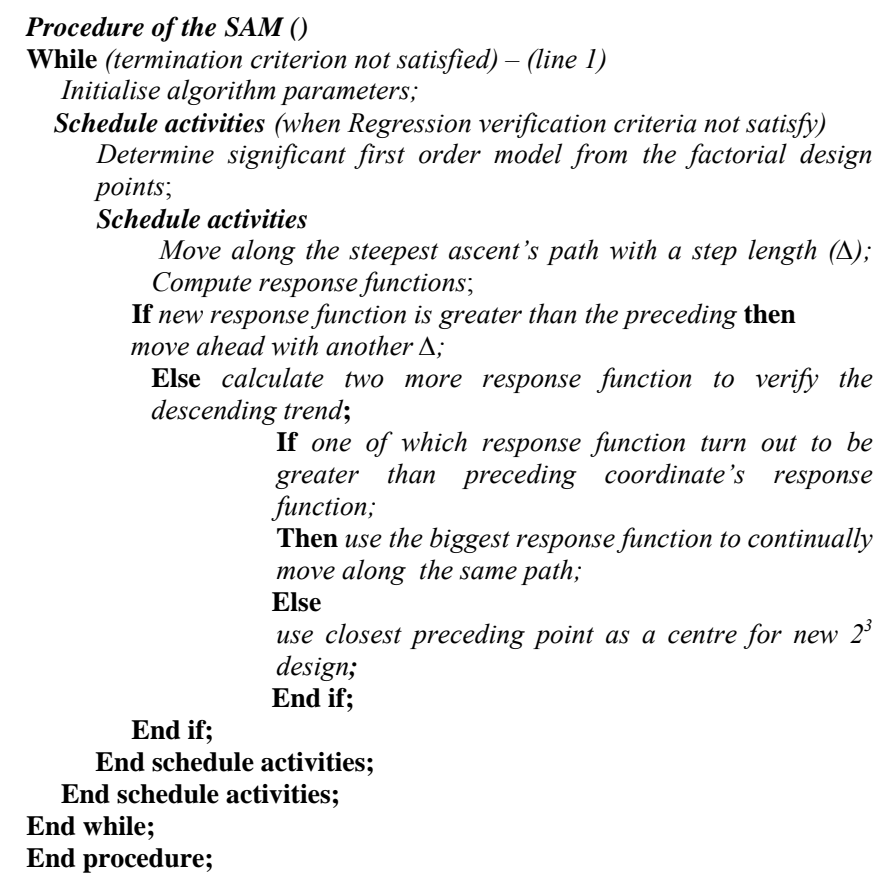

Figure 1. Pseudo Code of the SAM.

\section{B. Simulated Annealing (SA)}

Simulated Annealing has been derived from an interesting analogy between problems in statistical mechanics and multivariate or combinatorial optimisation [13]. This algorithm is a set of rules for searching large solution spaces in a manner that mimics the annealing process of metals. The algorithm simulates the behavior of an ensemble of atoms in equilibrium at a given finite temperature and its original framework can be traced to Metropolis [14]. This algorithm has been regularly used in global function optimisation and statistical applications.

In case of maximisation the procedures of this algorithm start at a corresponding initial value of the objective function, $y_{0}$. The new objective value, $y_{1}$, will be then determined. The new solution will be unconditionally accepted if its objective value is improved and the process regularly continues. Otherwise the difference or size of increment in objective values, $\Delta y$, is calculated and with an auxiliary experiment the new solution would be accepted with probability $\mathrm{P}(\Delta y)$ given by:

$$
\begin{aligned}
\mathrm{P}(\Delta y) & =1 & & \text { if } \Delta y=y_{1}-y_{0} \geq 0 \\
& =\operatorname{EXP}\left(c y_{0}{ }^{g} \Delta y\right) & & \text { if } \Delta y<0,
\end{aligned}
$$

where $c$ and $g$ are an arbitrary positive number and a negative number respectively. A random number, $x$, is generated from the uniform distribution on $(0,1)$ and is compared to $\operatorname{EXP}\left(c y_{0}{ }^{g} \Delta y\right)$. If $x<\operatorname{EXP}\left(c y_{0}{ }^{g} \Delta y\right)$, then the new solution is accepted. Otherwise it is rejected. This stochastic element is from Monte Carlo sampling. It occasionally allows the algorithm to accept the new solution to the problems, which deteriorate rather than improve the objective function value. However, Simulated Annealing includes a number of parameters including $g$ and $c$ they have been claimed that affect the efficiency of the algorithm. The pseudo code is used to briefly explain to all the procedures of the SA shown in Figure 2.

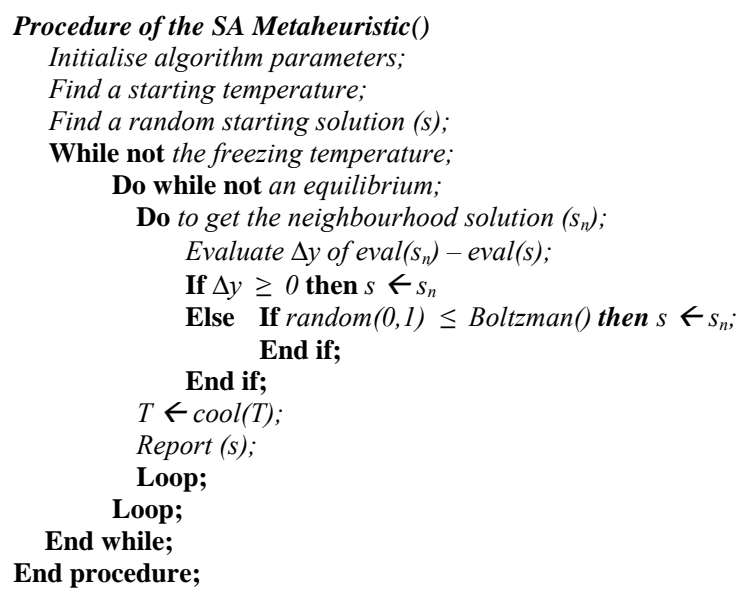

Figure 2. Pseudo Code of the SA Metaheuristic.

\section{Firefly Algorithm (FFA)}

The firefly algorithm (FFA) is a metaheuristic algorithm, inspired by the flashing behaviour of fireflies. The primary purpose for a firefly's flash is to act as a signal system to attract other fireflies. Now this can idealise some of the flashing characteristics of fireflies so as to consequently develop firefly-inspired algorithms. For simplicity in describing our new Firefly Algorithm, there are the following three idealised rules. 
On the first rule, each firefly attracts all the other fireflies with weaker flashes. All fireflies are unisex so that one firefly will be attracted to other fireflies regardless of their sex. Secondly, attractiveness is proportional to their brightness which is reverse proportional to their distances. For any two flashing fireflies, the less bright one will move towards the brighter one. The attractiveness is proportional to the brightness and they both decrease as their distance increases. If there is no brighter one than a particular firefly, it will move randomly. Finally, no firefly can attract the brightest firefly and it moves randomly.

The brightness of a firefly is affected or determined by the landscape of the objective function. For a maximisation problem, the brightness can simply be proportional to the value of the objective function. Other forms of brightness can be defined in a similar way to the fitness function in genetic algorithms. Based on these three rules, the basic steps of the firefly algorithm (FFA) can be summarised as the pseudo code shown in Figure 3.

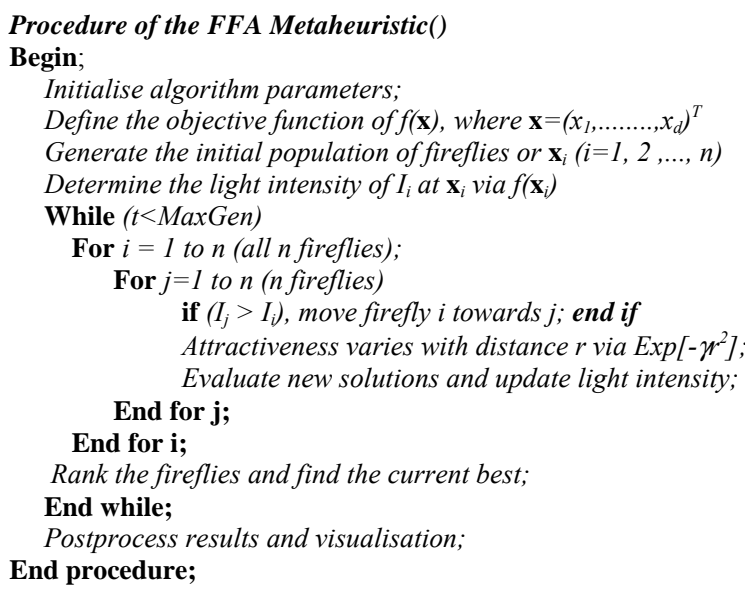

Figure 3. Pseudo code of the FFA Metaheuristic.

\section{Ant Colony Optimisation (ACO)}

Ant algorithm was first proposed by Dorigo and his colleagues as a multi-agent approach to optimisation problems, such as a travelling salesman problem (TSP) and a quadratic assignment problem (QAP). There is currently a lot of ongoing activity in the scientific community to extend or apply ant-based algorithms to many different discrete optimisation problems. Recent applications cover problems like a vehicle routing, a plant layout and so on. Ant algorithm is inspired by observations of real ant colonies. Ants are social insects and they live in colonies. Behaviour is direct more to the survival of the colony as a whole than to that of a single individual component of the colony. Social insects have captured the attention from many scientists because of a structure of their colonies, especially when compared with a relative simplicity of the colony's individual. An important and interesting behaviour of ant colonies is their foraging behaviour and in particular how ants can find shortest paths between food sources and their nest.

While walking from food sources to the nest and vice versa, ants deposit on the ground a substance called pheromone, forming a pheromone trail. With ants ability to smell pheromone they tend to choose a path marked by strong pheromone concentrations with the higher probability. The pheromone trail allows the ants to find their way back to the food source and vice versa. It can be also used by other ants to find the location of the food sources found by their nest mates. The pseudo code is used to briefly explain to all the procedures of the ACO shown in Figure 4.

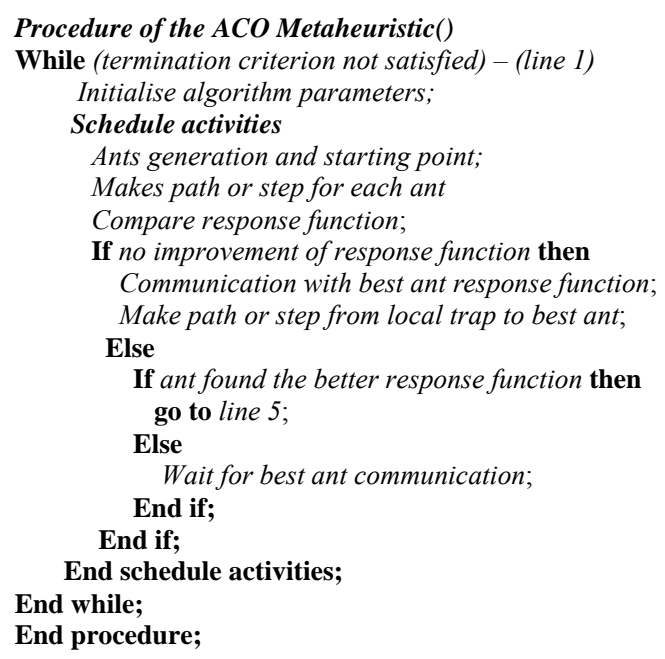

Figure 4. Pseudo code of the ACO Metaheuristic.

\section{Continuous StirRed TANK ReActor (CSTR)}

A diagrammatic representation of a single continuous stirred tank reactor (CSTR) is shown in Figure 5. For the CSTR a stream rich in chemical A of feed concentration $C_{A(i n)}$ is flowing into a reactor at a feed flow rate of $F_{(i n)}$, and a feed temperature of $T_{(i n)}$. The reaction in the CSTR is an irreversible, first order exothermic reaction. The proportion of chemical $\mathrm{A}$ is converted to a desired product $\mathrm{B}$, which, in turn, at high temperature undergoes further reaction and is decomposed to form an undesired by-product $\mathrm{C}$. The stated objective is to explore the operating conditions corresponding to higher concentration of product.

It is also assumed that level is perfectly controlled, so the volume of material in the tank is constant. This implies that the flow out equals the flow in. The temperature in the reactor may be regulated by manipulating the flow rate of the cooling water $\left(\mathrm{F}_{\mathrm{C}}\right)$ in the heat exchanger by the following control algorithm.

$$
\mathrm{F}_{\mathrm{C}}=\mathrm{T}_{\mathrm{BIAS}}-\mathrm{K}_{\mathrm{c}}\left(\mathrm{T}_{\mathrm{r}}-\mathrm{T}\right)
$$

A mechanistic model adequately accounting for the system under study is suggest purely by physical consideration and the dynamics of the system can then be described by the following set of ordinary, non-linear differential equations.

$$
\begin{aligned}
& V \frac{d C_{A}}{d t}=-K_{O A} e^{\left(-E_{A} / R T\right)} C_{A} V+F_{(i n)}\left(C_{A(i n)}-C_{A}\right) \\
& V \frac{d C_{B}}{d t}= K_{O A} e^{\left(-E_{A} / R T\right)} C_{A} V-K_{O B} e^{\left(-E_{B} / R T\right)} C_{B} V+F_{(i n)}\left(-C_{B}\right) \\
& \rho c_{p} V \frac{d T}{d t}=\left(-\Delta H_{R A}\right) K_{O A} e^{\left(-E_{A} / R T\right)} C_{A} V+\left(-\Delta H_{R B}\right) K_{O B} e^{\left(-E_{B} / R T\right)} C_{B} V \\
&+\rho c_{p} F_{(i n)}\left(T_{(i n)}-T\right)+\rho c_{p} F_{R}\left(T_{R}-T\right) \\
& \rho c_{p} V_{R} \frac{d T_{R}}{d t}=\rho c_{p} F_{R}\left(T-T_{R}\right)+U A\left(T_{C}-T_{R}\right) \\
& \rho c_{p} V_{C} \frac{d T_{C}}{d t}=\rho c_{p} F_{C}\left(T_{c(i n)}-T_{C}\right)+U A\left(T_{R}-T_{C}\right)
\end{aligned}
$$


The five state variables, which depend on time $t$, are the concentration of reactant $C_{A}$, the concentration of product $C_{B}$, the reactor temperature of $\mathrm{T}$, the temperature of the recycled flow of $T_{R}$ and the temperature of the coolant leaving the heat exchanger $\left(\mathrm{T}_{\mathrm{C}}\right)$. Initial conditions, values of the parameters of the process and parameters of the feedback controller, which determines the flow of coolant, are given in Appendix. The time constant of the system is such that equilibrium is attained after approximately ten minutes.

There are three predictor variables, which can be set to any chosen values within safe limits. These predictor variables relate to the feed flow are shown in Table I. The response variable of the process is defined to be the concentration of the desired product $\mathrm{B}, \mathrm{C}_{\mathrm{B}}$.

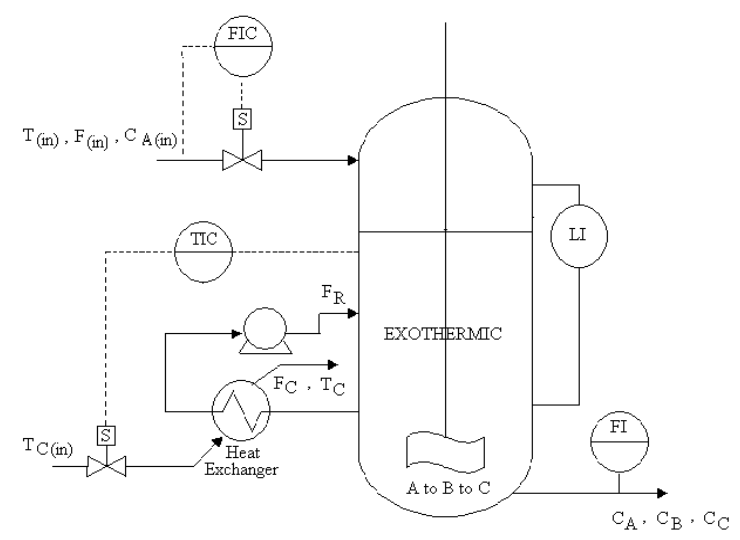

Figure 5. The Continuous Stirred Tank Reactor.

TABLE I. PREDICTOR VARIABLES OF FEED FLOW AND THEIR SAFE LIMITS

\begin{tabular}{|c|c|c|c|}
\hline $\begin{array}{c}\text { Predictor } \\
\text { Variable }\end{array}$ & Description & Unit & $\begin{array}{c}\text { Feasible } \\
\text { Region }\end{array}$ \\
\hline$T_{(i n)}$ & $\begin{array}{c}\text { Feed temperature of } \\
\text { reactant A }\end{array}$ & Celsius & $60-100$ \\
\hline$F_{(i n)}$ & Feed flow rate of reactant A & Liter/min & $1-10$ \\
\hline$C_{A(i n)}$ & Concentration of reactant A & Mole/liter & $1-15$ \\
\hline
\end{tabular}

\section{PARAmeter Settings For The Proposed METAHEURISTICS}

The parameters of the proposed methods, SA, FFA and ACO are varied in a factorial design. For the SA, the levels of $g$ and $c$ were selected to cover the range of values commonly found in the literature: $[-0.5,-1,-1.5]$ and $[4.5,6.5]$ for $g$ and $c$, respectively. Two replicates were performed for each of the 6 sets of parameter values. Each trial used the random initial design points, evenly distributed about edges, furthest from the optimum, of the safe region of operation. The comparisons were made for four different levels of measurement noise added to the response: independent and normally distributed with mean of zero and standard deviations of $0.5,1.0,2.0$ and 3.0 , respectively. The following performance measures on the yield at the end of each trial were considered.

\section{A. Taguchi's Measure of Performance $\left(Y_{l}\right)$}

Taguchi [15] proposed 'the larger the better' measure:

$$
Y_{1}=-10 \log \left(\sum_{i=1}^{n}\left(1 / y_{i}^{2}\right) / n\right),
$$

in which $y_{i}$ represents the highest yield at the end of trial $i$, and $n$ is the number of trials.

\section{B. Minimax Performance Measure $\left(Y_{2}\right)$}

Another measure of the performance of the approaches is the minimum of the highest yields at the end of the trials. In the case of ten trials, for example,

$$
Y_{2}=\operatorname{Min}\left(y_{1}, y_{2}, \ldots, y_{10}\right) \text {. }
$$

We wish to maximise $Y_{2}$.

\section{Mean Squared Error Performance Measure ( $\left.Y_{3}\right)$}

It is natural to consider combing bias and variance through the mean squared error (MSE) criterion [16]. In this case, for example,

$$
Y_{3}=\left[\left(\omega_{\mu}-\mathrm{T}\right)^{2}+\omega_{\sigma}^{2}\right]
$$

in which $\omega_{\mu}$ represents the average of actual responses, T represents the target value of response and $\omega_{\sigma}$ is the standard deviation of actual responses. We wish to minimise $Y_{3}$.

A typical table of results of the analysis of variance from the SA is given in Table II and the main effect plot with the error standard deviation of 1.0 for $Y_{1}$ is shown in Table III and Figure 6.

TABLE II. ANOVA FOR $\mathrm{Y}_{1}$ OF THE SA

\begin{tabular}{|c|c|c|c|c|c|}
\hline $\begin{array}{c}\text { Source of } \\
\text { Variation }\end{array}$ & $\begin{array}{c}\text { Sum of } \\
\text { Square }\end{array}$ & $\begin{array}{c}\text { Degree of } \\
\text { Freedom }\end{array}$ & $\begin{array}{c}\text { Mean } \\
\text { Square }\end{array}$ & $\boldsymbol{F}$ & P-Value \\
\hline$g$ & 0.0579 & 2 & 0.0289 & 0.44 & 0.665 \\
\hline$c$ & 0.7161 & 1 & 0.7161 & 10.81 & 0.017 \\
\hline$g^{*} c$ & 0.3667 & 2 & 0.1833 & 0.0663 & 0.141 \\
\hline Error & 0.3977 & 6 & 0.0663 & & \\
\hline Total & 1.5384 & 11 & & & \\
\hline
\end{tabular}

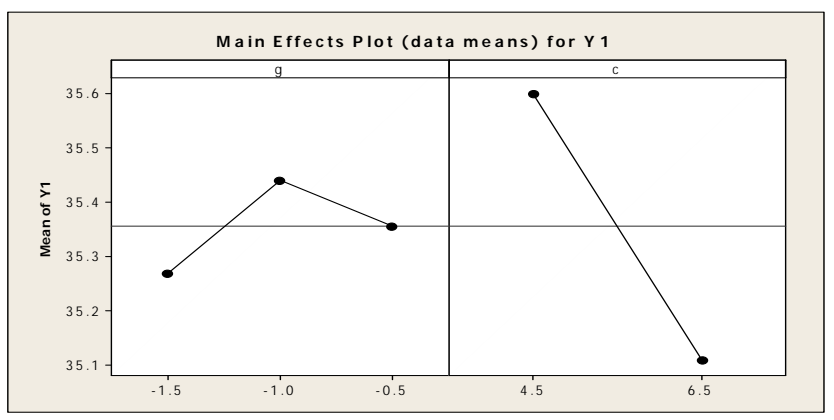

Figure 6. Main Effect Plots for $Y_{1}$ with the Error Standard Deviation of 1.0 .

With the error standard deviation (Stdev.) of 1.0, the main finding was that the probability of $g$ for $Y_{1}$ and $Y_{3}$ should be high $(-0.5)$. This leads to higher average and lower level of standard deviation of actual responses. No other statistically significant results were found. The preferred levels of $g$ and $c$ could be high ( -0.5 or -1.0$)$ and low (4.5), respectively. Results are included for all cases in which the ANOVA $\mathrm{P}$-values, for main effects and interaction, are less than 0.1 in Table III.

TABLE III. THE PREFERRED LEVELS OF THE PARAMETERS OF THE SA

\begin{tabular}{|c|c|c|c|c|c|}
\hline \multirow{2}{*}{ Stdev. } & \multicolumn{2}{|c|}{ Preferred Levels } & \multicolumn{3}{c|}{$\begin{array}{c}\text { Overall Significant and } \\
\text { P-Value }\end{array}$} \\
\cline { 2 - 6 } & $g$ & $c$ & $Y_{1}$ & $Y_{2}$ & $Y_{3}$ \\
\hline 0.5 & -0.5 & - & 0.081 & - & 0.077 \\
\hline 1.0 & -1.0 & 4.5 & 0.017 & - & - \\
\hline & - & 4.5 & - & 0.048 & - \\
\hline & $-1,-0.5$ & 4.5 & - & - & 0.040 \\
\hline 2.0 & - & - & - & - & - \\
\hline 3.0 & - & - & - & - & - \\
\hline
\end{tabular}

Similarly, preferable levels of parameters are determined and are set to be suggested levels for SA, FFA and ACO parameters (Table IV). Under a consideration of 
recommended levels of their parameters, those may bring the benefit to solve the CSTR.

TABLE IV. THE Preferred LEVELS OF THE PARAMETERS OF THE SA FFA AND ACO.

\begin{tabular}{|c|c|c|c|c|c|c|c|c|}
\hline \multirow{2}{*}{ Stdev. } & \multicolumn{2}{|c|}{ SA } & \multicolumn{2}{c|}{ FFA } & \multicolumn{4}{c|}{ ACO } \\
\cline { 2 - 9 } & $g$ & $c$ & $\beta_{0}$ & $\gamma$ & $\rho$ & $\eta$ & $\xi$ & $\delta$ \\
\hline 0.05 & -0.5 & 4.5 & 1 & 0.01 & 0.6 & 0.1 & 0.1 & 1.5 \\
\hline 1.0 & -1.0 & 4.5 & 1 & 0.01 & 0.4 & 0.5 & 0.5 & 2.5 \\
\hline 2.0 & -0.5 & 4.5 & 1 & 0.50 & 0.4 & 0.5 & 0.5 & 2.5 \\
\hline 3.0 & -0.5 & 4.5 & 1 & 0.50 & 0.4 & 0.5 & 0.5 & 2.5 \\
\hline
\end{tabular}

\section{Details of the Proposed Methods}

SAM Parameters: the volume of the factorial design [8]; the step length [1]; the significance level for tests of significance of slopes [10\%].

$S A$ Parameters: arbitrary positive and negative numbers of $g$ [-1] and $c[4.5]$.

FFA Parameters: the attractiveness at $r=0$ of $\beta_{0}$ [1]; the light absorption coefficient of $\gamma[0.01]$.

ACO Parameters: pheromone evaporation rate of $\rho[0.4]$; heuristic factor for preferring among available options of $\eta$ $[0.5] ; \xi$ and $\delta$ are exponent parameters that control the relative importance of pheromone concentration versus the heuristic factor [0.5 and 2.5].

Step 1: Perform a $2^{3}$ design at a random centre point.

Step 2: Fit a regression plane to the data so that the fitted model has the form

$$
\hat{y}=\hat{\beta}_{0}+\hat{\beta}_{1} T_{(i n)}+\hat{\beta}_{2} F_{(i n)}+\hat{\beta}_{3} C_{A(i n)} .
$$

Step 3: Test whether there is evidence that either $\beta_{1}, \beta_{2}$ or $\beta_{3}$ is different from zero at the $10 \%$ level of significance, i.e. does $\left|\frac{\hat{\beta}_{i}}{\sqrt{C_{i i} M S E}}\right|$ exceed $t_{v, 0.10}$ ?, where $v$ is the number of degree of freedom, one for the first experiment increasing by eight for each replicated experiment.

Step 4a: If the result is significant, move one step along the path of steepest ascent, that is along the line whose formula in parametric form is (f $\left.\hat{\beta}_{1}, \mathrm{f} \hat{\beta}_{2}, \mathrm{f} \hat{\beta}_{3}\right),(-\infty<\mathrm{f}<\infty)$, and determine the yield. The step length is

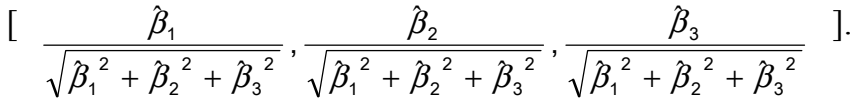

Otherwise go to Step $4 b$.

Step $4 b$ : Test whether there is evidence that the interaction or curvature check is significant. If the check is significant, go to Step 6. Otherwise, replicate the design and return to Step 2. Step 5a: If the yield is greater than the previous yield or the stochastic element meets the requirement of acceptance, continue by moving another step in the same direction.

Step $5 b$ : If the yield is not greater than the previous one, then calculate the objective increment $(\Delta y)$ and test the element as follows: Randomly generate a random variable, $x \sim$ Uniform $(0,1)$. Apply the following rules then go to Step $5 a$.

$$
\text { SA element: If } x<\mathrm{P}(\Delta y) \equiv \operatorname{EXP}\left(c y_{0}{ }^{g} \Delta y\right)
$$

FFA element: $\quad$ If $\left.x<\mathrm{P}(\Delta y) \equiv \beta_{0} \operatorname{EXP}\left[\gamma(\Delta y)^{2}\right)\right]$

$$
\begin{gathered}
\text { ACO element: If } x<\mathrm{P}(\Delta y) \equiv \frac{\tau_{i}^{\xi} \eta^{\delta}}{\tau_{i}^{\xi} \eta^{\delta}+\tau_{i-1}^{\xi} \eta^{\delta}} \\
\tau_{i}=\rho \tau_{i-1}+\Delta \tau
\end{gathered}
$$

; where $i$ is the number of iteration, $\rho \tau_{i-1}$ is the concentration of the pheromone at the previous iteration and $\Delta \tau$ is the cumulative process yields of the previous and current iterations.

Step 5c: Otherwise return to the preceding point then carry out another $2^{3}$ design and return to Step 2. If the first step leads to a yield less than the yields obtained in the preceding $2^{3}$ designs then replicate the design and go to Step 2 .

Step 6: Implement the finishing strategy. This is a central composite design (CCD) centred on the point $\left(T_{(i n) p}, F_{(i n) p}\right.$, $\left.C_{A(i n) p}\right)$, and fit then a quadratic surface to find the maximum $\left(T_{(i n) p}, F_{(i n) p}, C_{A(i n) p}\right)$. If $\left(T_{(i n) p}, F_{(i n) p}, C_{A(i n) p}\right)$ is within the volume of the designs, then $\left(T_{(i n) p}, F_{(i n) p}, C_{A(i n) p}\right)$ is taken as the optimum operating condition. If $\left(T_{(i n) p}, F_{(i n) p}, C_{A(i n) p}\right)$ is not within this volume, another CCD is carried out, centered on the point from the first CCD with the greatest yield. A quadratic surface is now fitted to all the data. If the maximum is outside the volume of the union of the two containing cubes, the ridge is searched for the greatest value of the function, using a step length of 0.05 (from additional experiments by using fewer runs).

\section{EXPERIMENTAL RESUlTS AND DISCUSSIONS}

In this work, for the computational procedures described above a computer simulation was implemented in Matlab 2006v.7.3B. A Laptop computer Lenovo R61 with Microsoft Windows version 5.1 (Build 2600.xpsp_sp2_gdr.070227-2254: service pack 2) was used for computational experiments. The comparison is made with the measurement noise on the concentration of the desired product B (normal and independent with zero mean and standard deviation of $0.5,1,2$ and 3 ). The typical three-dimensional response surfaces, with CA(in) fixed at 1 and 15, are shown in Figure 7. There are four performance measures over 50 realisations in this study. The first and second measures are an average $\left(\mathrm{Y}_{\mathrm{F} 1}\right)$ and a standard deviation $\left(\mathrm{Y}_{\mathrm{F} 2}\right)$ of greatest actual concentration of the desired product $\mathrm{B}$ from the finishing strategy respectively. The third is an average number of runs until the algorithms converge $\left(\mathrm{Y}_{\mathrm{F} 3}\right)$. Finally it is the percentage of sequences ended at the optimum $\left(\mathrm{Y}_{\mathrm{F} 4}\right)$.

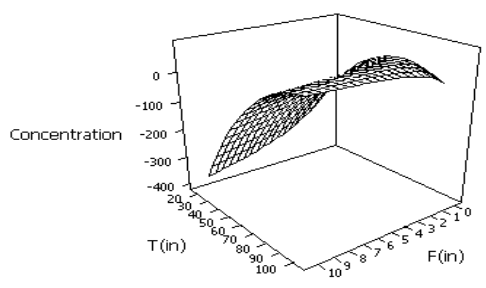

(a) 


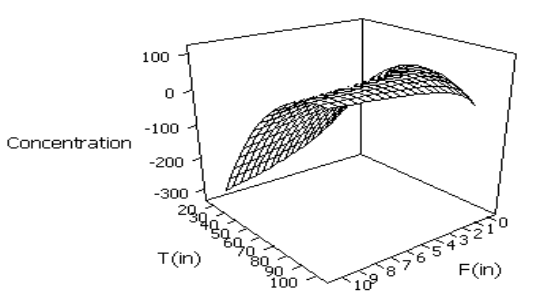

(b)

Figure 7. The Surface Plot with $C_{A(i n)}$ Fixed at 1 (a) and 15 (b) Respectively.

The process settings for all the scenarios are given in Table $\mathrm{V}$. The experimental results suggested that the conventional algorithm of SAM alone can produce an acceptable solution or even an optimal solution if the problem was not so noisy. When the problem is noisier, the integrated algorithms of SAM with SA (SAMSA), FFA (SAMFFA) and SAM with ACO (SAMACO) are more suitable to exploit a solution space as a local search by embedding within the SAM. The exploitation process can be performed on each population member to improve its experience and thus obtain a population of local optimum solutions. However, ant colony optimisation algorithm (ACO) requires higher levels of design points towards the optimum.

TABLE V. FOUR ACHIEVEMENTS OVER 50 REALISATIONS

\begin{tabular}{|c|c|c|c|c|}
\hline \multirow{2}{*}{$\begin{array}{c}\text { Performance } \\
\text { Measures }\end{array}$} & \multicolumn{4}{|c|}{ Standard Deviation of Noise } \\
\cline { 2 - 5 } & $\mathbf{0 . 5}$ & $\mathbf{1}$ & $\mathbf{2}$ & $\mathbf{3}$ \\
\hline \multirow{3}{*}{$\mathrm{Y}_{\mathrm{F} 1}$} & $59.3025^{\mathrm{A}}$ & 57.2845 & 59.3670 & 60.0308 \\
& $57.6587^{\mathrm{B}}$ & 57.4589 & 60.9887 & 60.5890 \\
& $56.7503^{\mathrm{C}}$ & 57.6083 & 61.6109 & 61.6276 \\
& $57.7124^{\mathrm{D}}$ & 57.9102 & 61.5745 & 61.0987 \\
\hline \multirow{3}{*}{$\mathrm{Y}_{\mathrm{F} 2}$} & 7.6283 & 7.3860 & 9.5658 & 7.7273 \\
& 10.9875 & 6.9987 & 7.0548 & 6.7858 \\
& 10.2718 & 6.8989 & 7.6716 & 6.4331 \\
& 11.3545 & 7.0215 & 7.8541 & 6.1568 \\
\hline & 33.03 & 33.57 & 32.06 & 31.53 \\
$\mathrm{Y}_{\mathrm{F} 3}$ & 49.01 & 35.26 & 35.98 & 36.02 \\
& 38.17 & 34.90 & 34.75 & 33.30 \\
& 40.25 & 35.69 & 36.25 & 35.95 \\
\hline & 0.85 & 0.87 & 0.82 & 0.78 \\
$\mathrm{Y}_{\mathrm{F} 4}$ & 0.84 & 0.87 & 0.89 & 0.86 \\
& 0.85 & 0.90 & 0.90 & 0.92 \\
& 0.83 & 0.89 & 0.89 & 0.85 \\
\hline
\end{tabular}

NoTE: SAM $^{\mathrm{A}}, \mathrm{SAMSA}^{\mathrm{B}}, \mathrm{SAMFFA}^{\mathrm{C}}, \mathrm{SAMACO}^{\mathrm{D}}$

In Figure 8, the actual yield and its best so far achieved on one replicate of the sequential procedures revealed the higher performance of all the integrated algorithms SAMSA, SAMFFA and SAMACO when compared with the conventional steepest ascent method (SAM). Accordingly to noises, some operating points from factorial designs may need to duplicate and measure process yields.

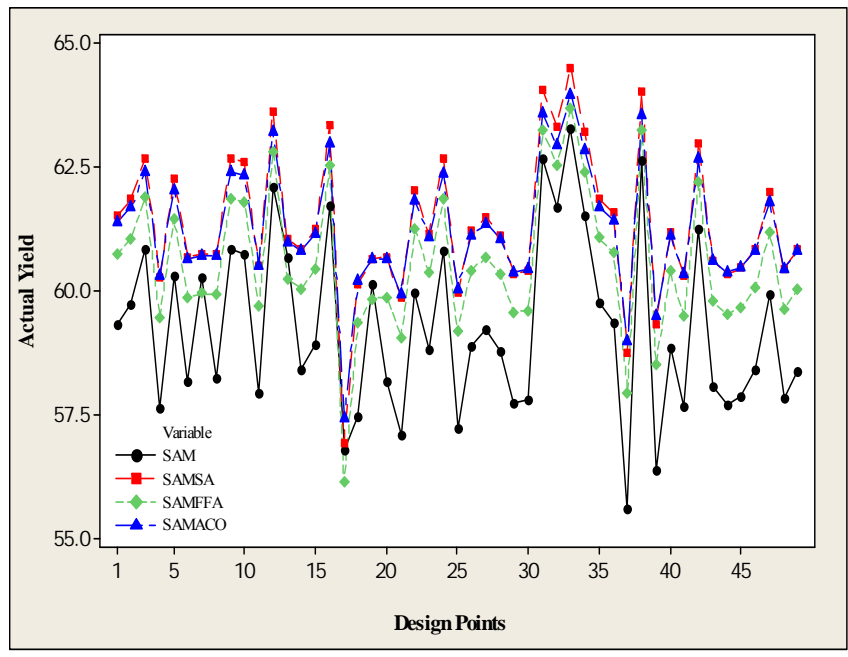

Figure 8. Sequential Performance of all Algorithms on the CSTR with Noise Standard Deviation of 2.0

The performance of the method of steepest ascent and the integrated approaches for 50 realisations can be explained by the Box-Whisker plots in Figure 9 when the error standard deviation levels were 2.0 and 3.0. Note that since the efficiency of these algorithms is related to their initial design points based on factorial designs, it would be helpful to set random starting points for all algorithms. These results show that the performance of the integrated approach under the stochastic elements of SA, FFA and ACO seems superior to the algorithm based on the method of steepest ascent at the higher levels of error standard deviations.

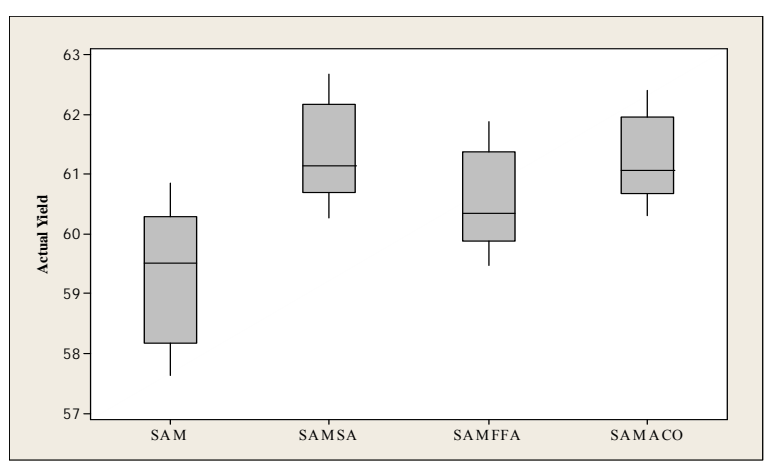

(a)

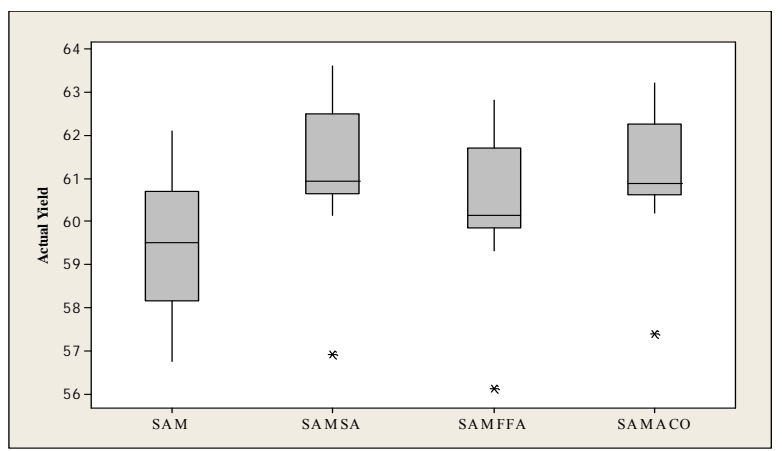

(b)

Figure 9. Two Independent Box-Whisker Plot Comparisons Showing the Performance of the SA and the Integrated Approaches when the Error Standard Deviation was 2.0 (a) and 3.0 (b) respectively.

An analysis of variance, ANOVA, is a confirmation 
technique for analysing experimental data in which a response or the actual yield is measured under various conditions identified by the algorithms. It can also be seen that these experimental results on all scenarios categorised by SAM, SAMSA, SAMFFA and SAMACO, were statistically significant with a $90 \%$ confidence interval (Tables VI and Figure 10). The numerical results suggested that all the hybridisations provided the better performance in terms of the average actual yield or the concentration of the desired product $\mathrm{B}, \mathrm{C}_{\mathrm{B}}$. The goodness of the linear statistical model via experimental errors or residuals is also adequate. As the results, all hybridisations seem to be better to apply to the CSTR, especially the hybridisations of SAM with SA and ACO.

TABLE VI. ONE-WAY ANOVA: ACTUAL YIELD VERRSUS FOUR SCENARIOS

\begin{tabular}{|c|c|c|c|c|c|}
\hline $\begin{array}{c}\text { Source of } \\
\text { Variation }\end{array}$ & $\begin{array}{c}\text { Sum of } \\
\text { Square }\end{array}$ & $\begin{array}{c}\text { Degree of } \\
\text { Freedom }\end{array}$ & $\begin{array}{c}\text { Mean } \\
\text { Square }\end{array}$ & F & P-Value \\
\hline Factor & 135.83 & 3 & 45.28 & 20.86 & 0.000 \\
\hline Error & 425.50 & 196 & 2.17 & & \\
\hline Total & 561.33 & 199 & & & \\
\hline
\end{tabular}

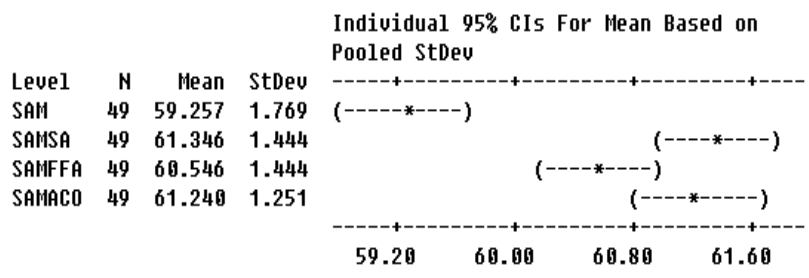

Figure 10. Graphical Comparison for Four Scenarios on the CSTR with Noise Standard Deviation of 2.0.

Moreover, the percentage of sequences ended at the optimum or near optimum of radius equalling two from the integrated approaches is better at higher levels of error standard deviation although the greater number of runs were required to converge to the optimum. To find out the best solution within a limited time, is obviously difficult. There are various modifications to overcome in the research. However, convergence to the global optimum is not substantially rapid.

Recommendations should be made for the values of the algorithm parameters, although these values depend on the selected performance measure. As stated earlier, the system in this research was restricted to three predictor variable. Consequently, comparisons and conclusions among the three algorithms may not be valid for other families of systems. Other stochastic approaches could be extended to the method based on conventional factorial designs to increase its performance, especially in terms of speed of convergence, when the error standard deviation is at higher levels.

\section{APPENDIX}

Initial conditions of process state variables, values of the parameters of the process and parameters of controller are given in Tables VII, VIII and IX, respectively. The time constant of the system is such that equilibrium is attained after approximately ten minutes.
TABLE VII. PROCESS STATE VARIABLES AND THEIR INITIAL CONDITIONS

\begin{tabular}{|c|l|c|c|}
\hline Variable & \multicolumn{1}{|c|}{ Description } & Unit & Value \\
\hline $\mathrm{C}_{\mathrm{A}}$ & Concentration of reactant A & $\mathrm{mole} / \mathrm{m}^{3}$ & 747.9 \\
\hline $\mathrm{C}_{\mathrm{B}}$ & Concentration of product $\mathrm{B}$ & $\mathrm{mole} / \mathrm{m}^{3}$ & 1609 \\
\hline $\mathrm{T}$ & Reactor temperature & $\mathrm{K}$ & 341.4 \\
\hline $\mathrm{T}_{\mathrm{R}}$ & Temperature of the recycled flow & $\mathrm{K}$ & 333.3 \\
\hline $\mathrm{T}_{\mathrm{C}}$ & Temperature of the coolant & $\mathrm{K}$ & 330.5 \\
\hline
\end{tabular}

TABLE VIII. PARAMETERS OF THE PROCESS

\begin{tabular}{|c|l|c|c|}
\hline Parameter & \multicolumn{1}{|c|}{ Description } & Unit & Value \\
\hline $\mathrm{V}$ & Volume of the CSTR & $\mathrm{m}^{3}$ & 3 \\
\hline $\mathrm{K}_{\mathrm{OA}}$ & Rate coefficient (A to B) & $\mathrm{mole} / \mathrm{s}$ & $7.10^{11}$ \\
\hline $\mathrm{E}_{\mathrm{A}}$ & Activation energy (A to B) & $\mathrm{J}$ & 90000 \\
\hline $\mathrm{R}$ & Gas constant & $\mathrm{J} / \mathrm{mole} / \mathrm{K}$ & 8.314 \\
\hline $\mathrm{K}_{\mathrm{OB}}$ & Rate coefficient (B to C) & $\mathrm{mole} / \mathrm{s}$ & $9.10^{11}$ \\
\hline $\mathrm{E}_{\mathrm{B}}$ & Activation energy (B to C) & $\mathrm{J}$ & 100000 \\
\hline$\rho$ & Process fluid density & $\mathrm{kg} / \mathrm{m}^{3}$ & 1000 \\
\hline $\mathrm{c}_{\mathrm{p}}$ & Process fluid heat capacity & $\mathrm{J} / \mathrm{kg} / \mathrm{K}$ & 4180 \\
\hline$-\Delta \mathrm{H}_{\mathrm{RA}}$ & Heat of reaction (A to B) & $\mathrm{J} / \mathrm{mole}$ & 80000 \\
\hline$-\Delta \mathrm{H}_{\mathrm{RB}}$ & Heat of reaction (B to C) & $\mathrm{J} / \mathrm{mole}$ & 40000 \\
\hline $\mathrm{F}_{\mathrm{R}}$ & Feed flow rate of recycled stream & $\mathrm{m}^{3} / \mathrm{s}$ & 0.025 \\
\hline $\mathrm{U}$ & Heat transfer coefficient & $\mathrm{W} / \mathrm{m}^{2} \mathrm{~K}$ & 3000 \\
\hline $\mathrm{A}_{\mathrm{V}}$ & Area, heat exchanger(HX) & $\mathrm{m}^{2}$ & 100 \\
\hline $\mathrm{V}_{\mathrm{C}}$ & Volume, cooling water in HX & $\mathrm{m}^{3}$ & 0.2 \\
\hline $\mathrm{V}_{\mathrm{R}}$ & Volume, process stream in HX & $\mathrm{m}^{3}$ & 0.2 \\
\hline $\mathrm{T}_{\mathrm{C}(\text { in) }}$ & Feed temperature of cooling stream & $\mathrm{K}$ & 293 \\
\hline
\end{tabular}

TABLE IX. PARAMETERS OF CONTROLLER

\begin{tabular}{|c|c|c|}
\hline Parameter & Description & Unit \\
\hline $\mathrm{T}_{\mathrm{r}}$ & Required temperature & $\mathrm{K}$ \\
\hline $\mathrm{K}_{\mathrm{C}}$ & Controller gain & $\mathrm{m}^{3} / \mathrm{sK}$ \\
\hline $\mathrm{T}_{\mathrm{BIAS}}$ & Offset & $\mathrm{m}^{3} / \mathrm{s}$ \\
\hline
\end{tabular}

\section{ACKNOWLEDGMENT}

An author gratefully acknowledges the computing assistance of P. Aungkulanon in the early phase of this research.

\section{REFERENCES}

[1] P. Luangpaiboon, "Hybridisations of simulated annealing and modified simplex algorithms on a path of steepest ascent with multi-response for optimal parameter settings of ACO," Proc. AIP Conference (ICOR2009), vol. 1174, Mar. 2009, pp. 94-108, doi: $10.1063 / 1.3256264$.

[2] E. Emad, H. Tarek and G. Donald, "Comparison among Five Evolutionary-based Optimisation Algorithms," Advanced Engineering Informatics, vol. 19, 2005, pp. 43-53, doi: 10.1016/j.aei.2005.01.004.

[3] S. Lukasik and S. Zak, "Firefly Algorithm for Continuous Constrained Optimisation Tasks", Lecture Notes in Computer Science, vol. 5796, 2009, pp. 97-106, doi:10.1007/978-3-642-04441-0_8.

[4] S. Häckel and P. Dippold, "The Bee Colony-inspired Algorithm (BCiA): A Two-stage Approach for Solving the Vehicle Routing Problem with Time Windows", Proc. Genetic and Evolutionary Computation Conference (GECCO-2009), 2009, pp. 25-32, doi: 10.1145/1569901.1569906.

[5] K.S. Lee and Z.W. Geem, "A New Metaheuristic Algorithm for Continuous Engineering Optimisation: Harmony Search Theory and Practice," Comput: Meth. Appl. Mech. Eng., vol. 194, 2004, pp. 3902-3933, doi: 10.1016/j.cma.2004.09.007 .

[6] Muller and D.R. Insua, "Issues in Bayesian Analysis of Neural Network Models,” Neural Computation, vol. 10, 1995, pp. 571-592, doi: 10.1162/089976698300017737.

[7] M. Dorigo, V. Maniezzo and A. Colorni, “Ant System: Optimisation by a Colony of Cooperating Agents," IEEE Transactions on Systems, Man, and Cybernetics Part B, vol. 26, numéro 1, 1996, pp. 29-41, doi: $10.1109 / 3477.484436$ 
[8] J.Y. Jeon, J.H. Kim and K. Koh, "Experimental Evolutionary Programming-based High-Precision Control," IEEE Control Sys. Tech., vol. 17, 1997, pp. 66-74, doi: 10.1109/37.581296.

[9] R. Storn, "System Design by Constraint Adaptation and Differential Evolution," IEEE Trans. on Evolutionary Computation, vol. 3, no. 1, 1999, pp. 22-34, doi: 10.1109/4235.752918

[10] M. Clerc and J. Kennedy, "The Particle Swarm-Explosion, Stability, and Convergence in a Multidimensional Complex Space," IEEE Transactions on Evolutionary Computation, vol. 6, 2002, pp.58-73, doi: $10.1109 / 4235.985692$.

[11] A. Lokketangen, K. Jornsten and S. Storoy, "Tabu Search within a Pivot and Complement Framework," International Transactions in Operations Research, vol. 1, no. 3, 1994, pp. 305-316, doi: 10.1111/1475-3995.d01-42.

[12] V. Granville, M. Krivanek and J.P. Rasson, "Simulated Annealing: a Proof of Convergence", Pattern Analysis and Machine Intelligence, IEEE Transactions, vol. 16, Issue 6, 1994, pp. 652 - 656, doi: $10.1109 / 34.295910$.

[13] S. Kirkpatrick, C.D. Jr. Gelatt and M.P. Vecchi, "Optimisation by Simulated Annealing", Science, vol. 220, 1983, pp. 671-680.

[14] I.O. Bohachevsky M.E. Johnson and M.L. Stein, "Generalised Simulated Annealing for Function Optimisation", Technometrics, vol. 28, no. 3, 1986, pp. 209-217.

[15] G. Taguchi and Y. Wu, Introduction to Off-Line Quality Control. Nagoya, Japan: Central Japan Quality Control Association, 1980.
[16] D.K.J. Lin and W. Tu, "Dual Response Surface Optimisation", Journal of Quality Technology, vol. 27, 1995, pp. 301-317.

P. Luangpaiboon has been a lecturer, and Associate Professor, in the Industrial Statistics and Operational Research Unit (ISO-RU), the department of Industrial Engineering at Thammasat University, THAILAND since 1995. He graduated his Bachelor (1989-1993) and Master Degrees (1993-1995) in Industrial Engineering from Kasetsart University, THAILAND and Ph. D. (1997-2000) in Engineering Mathematics from Newcastle upon Tyne, ENGLAND. He is a member of International Association of Computer Science and Information Technology (IACSIT) and International Association of Engineers (IAENG).

His research interests consist of meta-heuristics, optimisation, industrial statistics, the design and analysis of experiments and response surface methodology. He received Kasetsart University Master Thesis Award in 1995 (Dynamic Process Layout Planning), Certificate of Merit for The 2009 IAENG International Conference on Operations Research (A Hybrid of Modified Simplex and Steepest Ascent Methods with Signal to Noise Ratio for Optimal Parameter Settings of ACO) and Best Paper Award for the Operations Research Network Conference 2010 (An Exploration of Bees Parameter Settings via Modified Simplex and Conventional Design of Experiments). His email address is lpongch@engr.tu.ac.th. 